\title{
Fuzzy optimization for portfolio selection based on Embedding Theorem in Fuzzy Normed Linear Spaces
}

\author{
Farnaz Solatikia', Erdem Kiliç², Gerhard Wilhelm Weber ${ }^{3}$ \\ ${ }^{1}$ Department of Engineering Science, Middle East Technical University, Ankara, Turkey, \\ solatikia.farnaz@metu.edu.tr (corresponding author) \\ ${ }^{2}$ Erdem Kiliç, Department of Economics, MEF University, Istanbul, Turkey, \\ kilice@mef.edu.tr \\ ${ }^{3}$ Gerhard Wilhelm Weber, Institute of Applied Mathematics, Middle East Technical University, Ankara, Turkey, \\ gweber@metu.edu.tr
}

Background: This paper generalizes the results of Embedding problem of Fuzzy Number Space and its extension into a Fuzzy Banach Space $C(\Omega) \times C(\Omega)$, where $C(\Omega)$ is the set of all real-valued continuous functions on an open set $\Omega$.

Objectives: The main idea behind our approach consists of taking advantage of interplays between fuzzy normed spaces and normed spaces in a way to get an equivalent stochastic program. This helps avoiding pitfalls due to severe oversimplification of the reality.

Method: The embedding theorem shows that the set of all fuzzy numbers can be embedded into a Fuzzy Banach space. Inspired by this embedding theorem, we propose a solution concept of fuzzy optimization problem which is obtained by applying the embedding function to the original fuzzy optimization problem.

Results: The proposed method is used to extend the classical Mean-Variance portfolio selection model into Mean Variance-Skewness model in fuzzy environment under the criteria on short and long term returns, liquidity and dividends. Conclusion: A fuzzy optimization problem can be transformed into a multiobjective optimization problem which can be solved by using interactive fuzzy decision making procedure. Investor preferences determine the optimal multiobjective solution according to alternative scenarios.

Keywords: Embedding problem, Fuzzy optimization, Fuzzy Banach Space, Portfolio selection.

\section{Introduction}

Based on the fact that in many situations the distance between two points is in exact rather than a single real number, Kaleva and Seikkala initiated the concept of a fuzzy metric space by describing the distance of points as $\mathrm{s}$ fuzzy real number. Since each usual metric space and each Menger probabilistic metric space can be considered as $\mathrm{s}$ special case of fuzzy metric space, the study for the fuzzy metric space has been attracted many authors, and several results for nonlinear mappings have been given in some literatures. The concept of a fuzzy norm on a linear space is of comparatively recent origin. It was Katsaras, who while studying fuzzy topological vector spaces, was the first to introduce in 1984 the idea of fuzzy norm on a linear space. Following his pioneering work, Felbin offered in 1992 an alternative definition of a fuzzy norm on a linear space with an associated metric of the Kaleva and Seikkala type. A further development along this line of inquiry took place when in 1994; Cheng and Mordeson evolved the definition

Received: $15^{\text {th }}$ January 2014; revised: $14^{\text {th }}$ February 2014; accepted $24^{\text {th }}$ March 2014 
of a further type of fuzzy norm having a corresponding metric of the Kramosil and Michalek type. Since these results apparently also constitute various types of fuzzy norms, the further line of inquiry that obviously suggests itself pertains to the determination of relations, if any, among these fuzzy norms. In 2007, an attempt was made to find such relation by making a comparative study of the fuzzy norms defined by Katsaras, Felbin and Bag and Samanta. It has been observed that the fuzzy norm defined by Bag and Samanta is similar to that of Katsaras who defined it in a different way. On the other hand, Felbin's type fuzzy norm corresponds to a pair of which one is a fuzzy norm in our sense and the other is a fuzzy anti-norm. It is as important the concept of Menger probabilistic normed linear space introduced by Serstnev and moreover, each usual classical normed space and Menger probabilistic normed spaces is special cases of fuzzy normed linear spaces. The authors (Sadeqi I, Solatikia F. 2010; Weber G.-W., Solatikia F. 2013) showed that each of the category of fuzzy normed space and Menger probabilistic normed space is isomorphically a subcategory of the category of topological vector spaces and all results and theorems of topological vector spaces apply to fuzzy normed space and Menger probabilistic normed space in general, also as a new result they showed that $C(\Omega)$, is a fuzzy normed space then Menger probabilistic normed space, while it is not classically norm able. So, if we replace the normed space $C[0,1]$ by the fuzzy normed linear space $C(\Omega)$, then we get an important result in fuzzy normed linear space, while the same statement does not hold true in classical analysis.

Portfolio optimization is the process of allocating budget between assets and managing the assets within it. The modern portfolio theory has been proposed by Markowitz (1952) that considers expected return and risk for a portfolio selection problem where variance is used as risk measure. Markowitz's mean-variance model is the central source for a single period portfolio selection problem, from where the efficient frontier model with beta market risk and risk free return can be exploited. As a common standard portfolio model it is used the determine the performance of financial investments and serves as a hedge instrument with encompassing specifications. A wide range of fuzzy model applications are derived from the initially proposed Markowitz model. Genetic algorithms with applications of portfolio selection models in a fuzzy framework are analyzed by Gupta et al. (2012), Bermúdeza et al. (2012), Li and Xu (2013), Khalili-Damghani et al. (2013). Gupta analyze a multiobjective credibilistic model with fuzzy constraints of the portfolio selection problem. A hybrid intelligent algorithm that integrates fuzzy simulation with a real-coded genetic algorithm is contructed. They claim for more efficiency where fuzzy parameters are characterized by general functional forms. Bermúdeza proposed genetic algorithms transfering applications from their traditional domain of optimization to fuzzy ranking strategy for selecting efficient portfolios of restricted cardinality. A special focus is put on the ambiguity of the trapezoidal fuzzy number which represents the uncertainty on the return. A hybrid multiobjective framework integrates and synthesizes a genetic based machine learning method to design an alternative fuzzy ranking system for comparison purposes based on the results from a data mining model with the results from a data envelope analysis model. Metaxiotis and Liagkouras (2012), and Khalili-Damghani et al. (2013) use evolutionary algorithms to evaluate fuzzy portfolio models. Metaxiotis and Liagkouras present a portfolio management model with the support of multiobjective evolutionary algorithms. They utilize this framework to gain an understanding of the current state of areas related to applications in the portfolio management research field. Khalili-Damghani develop an evolutionary algorithm to design the structure of fuzzy rule based multiobjective system. Tsaur (2013), Li and Xu (2013), Huang and Qiao (2012) put their focus on a behavioral aspect of portfolio selection. Investor behaviors can be classified as risk averse, risk neutral or risk affine. Tsaur conducts a behavioral analysis of the investor in the fuzzy portfolio model. They examine different investor risk preferences to discover an efficient method for fuzzy portfolio selection. In this relation, a probabilistic meanstandard deviation model is applied to fit the risk attitudes of investors. In an application of a multiobjective portfolio selection model with fuzzy random returns $\mathrm{Li}$ and $\mathrm{Xu}$ use experts' opinions and judgments and investors' different attitudes in the portfolio selection process, such that the investor's individual preference on return, risk and liquidity are considered by the help of a genetic algorithm. A similar model is proposed by Huang and Qiao to enable the users to solve a problem with currently available programming tools. In addition, a method of obtaining the uncertainty distributions of the security returns is described based on experts' evaluations and presented illustratively. A TOPSIS compromised programming approach is designed by Liu et al. (2012) to transform a portfolio selection problems in fuzzy environment into single objective models. The model includes return, transaction cost, risk and skewness of the portfolio as decision parameters.

Wu (2004) develops models with fuzzy space and special spaces such as Banach spaces. In particular, $\mathrm{Wu}$ and Ma (1991) provide a specific Banach space that the set of all fuzzy real numbers, which was introduced by Zadeh (1965) and plays the most fundamental role in the theory of fuzzy analysis, can be embedded into a Banach space $C[0,1]$ $\times C[0,1]$, where $C[0,1]$ is the set of all real-valued bounded functions on $[0,1]$ such that it is left-continuous for any $t \in$ $(0,1]$ and right-continuous at 0 , and has a right limit for any $t \in(0,1]$. In 2011, Bhattacharyya and Kar extend the classical Mean-Variance portfolio selection model into Mean Variance Skewness model in fuzzy environment under the criteria on short and long term returns, liquidity and dividends. The embedding theorem is used to convert the fuzzy MVS model into crisp multi-objective constrained 
optimization problem. They proposed a convenient tool for deep research of fuzzy analysis. It seems therefore reasonable to think if the concept of Fuzzy optimization problems based on the embedding theorem and also Multiobjective fuzzy optimization for portfolio selection can be extended to probabilistic normed spaces and in that case enquire how the basic properties are affected.

In this paper, the embedding theorem from the set of all fuzzy numbers into a Banach space $C[0,1] \times C[0,1]$, is extended to more general case, i.e., from fuzzy number space into a Fuzzy Banach space $C(\Omega)$, where $C(\Omega)$ is the set of all real-valued continuous functions on an open set $\Omega$, to propose a solution concept of fuzzy optimization problem which is obtained by applying the embedding function to the original fuzzy optimization problem.

\section{Preliminaries}

For readers convenience, in this section we briefly recall some concepts and results from Menger probabilistic normed spaces theory used in the paper.

Definition: A mapping $\eta: \mathfrak{R} \rightarrow[0,1]$ is called a fuzzy real number, whose $\alpha-$ level set is denoted by $[\eta]_{\alpha}$, i.e., $[\eta]_{\alpha}=\{t: \eta(t) \geq \alpha\}$, if it satisfies two axioms:

$(N 1)$ There exists $t_{0} \in \mathfrak{R}$ such that $\eta\left(t_{0}\right)=1$.

(N2) For each $\alpha \in(0,1]:[\eta]_{\alpha}=\left[\eta_{\alpha}^{-}, \eta_{\alpha}^{+}\right],-\infty<\eta_{\alpha}^{-} \leq \eta_{\alpha}^{+}<+\infty$.

The set of all fuzzy real numbers denoted by $\mathfrak{R}(I)$. If $\eta \in \mathfrak{R}(I)$ and $\eta(t)=0$ whenever $t<0$, then $\eta$ is called a non-negative fuzzy real number and $\mathfrak{R}^{*}(I)$ stands for the set of all non-negative fuzzy real numbers. The number 0 stands for the fuzzy number satisfying $0(t)=1$ if $t=0$ and $0(t)=0$ if $t \neq 0$ clearly $0 \in \mathfrak{R}^{*}(I)$. The set of all real numbers can be embedded in $\mathfrak{R}$ because if $r \in(-\infty,+\infty)$, then $r \in \mathfrak{R}(I)$ satisfies $r(t)=0(t-r)$. According to Puri and Ralescu in 1986, we define the metric $d_{\{F}$, when $\eta_{1}, \eta_{2} \in \mathfrak{R}(I)$ as: $\left.d_{\{F\}}\left(\left[\eta_{1}\right]_{\alpha},\left[\eta_{2}\right]_{\alpha}\right)=\sup _{0<\alpha<1} \max \left\{\left|\eta_{1\{\alpha\}}^{-}-\eta_{2\{\alpha\}}^{-}\right|, \mid \eta_{1\{\alpha\}}^{+}-\eta_{2\{\alpha\}}^{+}\right) \mid\right\}$.

Definition: Let $X$ be a real linear space; $L$ and $R$ be symmetric and non-decreasing mapping from $[0,1] \times[0,1]$ into $[0,1]$ satisfying $L(0,0)=0, R(1,1)=1$. Then $\|\cdot\|$ is called a fuzzy norm and $(X,\|\|, L, R$.$) a fuzzy normed$ linear space if $\|\|:. X \rightarrow \mathfrak{R}^{*}(I)$ satisfies the following axioms, where $[\|x\|]_{\alpha}=\left[\|x\|_{\alpha}^{-},\|x\|_{\alpha}^{+}\right]$for $x \in X$ and $\alpha \in(0,1]:$

(A) $1\|x\|=\overline{0}$ if and only if $x=0$,

(A)2 $\|\mathrm{r} x\|=\mid r\|x\|$ for all $x \in X$ and $r \in(-\infty, \infty)$,

(A)3 For all $x, y \in X$ :

$(A 3 L)$ whenever $s \leq\|x\|_{1}^{-}, t \leq\|x\|_{1}^{-}$and $s+t \leq\|x+y\|_{1}^{-}$, then $\|x+y\|(s+t) \leq R(\|x\|(s),\|y\|(t))$, $(A 3 R)$ whenever $s \geq\|x\|_{1}^{-}, t \geq\|y\|_{1}^{-}$and $s+t \geq\|x+y\|_{1}^{-}$, then $\|x+y\|(s+t) \leq R(\|x\|(s),\|y\|(t))$.

Definition: Let $X$ be a vector space. A fuzzy subset $N$ of $X \times \Re$ is called a fuzzy norm on $X$ if the following conditions:
N1) $N(x, t)=0 ; \forall t \in \mathfrak{R}$ with $t \leq 0$,

N2) $N(x, t)=1 ; \forall t \in \mathfrak{R}, t>0$ iff $x=0$,

N3) $N(c x, t)=N\left(x, \frac{t}{|c|}\right) ; \forall t \in \Re, t>0$ and $c \neq 0$,

N4) $N(x+y, t+s) \geq \min \{N(x, s), N(y, t)\}$; for all $x, y \in X$ for all $s, t \in \mathfrak{R}$,

N5) $N(x,$.$) is a non -decreasing function on \mathfrak{R}$, and $\lim _{t \rightarrow \infty} N(x, t)=1$.

The pair $(X, N)$ is said to be a fuzzy normed space.

Definition: Let $X$ and $Y$ be topological spaces. An embedding function of $X$ into $Y$ is a function $e: X \rightarrow Y$ which is a homeomorphism when considered as a function from $X$ onto $e(X)$. A function $e: X \rightarrow Y$ is an embedding function if and only if it is continuous and one-to-one and for every open set $V$ in $X$ there exists an open subset $W$ of $Y$ such that $e(V)$ is the intersection of $W$ and $Y$.

\section{Main results}

Theorem: Let $\left(X, N_{1}, *\right)$ and $\left(Y, N_{2}, *\right)$ be two fuzzy normed spaces. Let $(x, y) \in X \times Y, t \in \mathfrak{R}$, and $N((x, y), t)=\min \left\{N_{1}(x, t), N_{2}(y, t)\right\}$. Then $\left(X \times Y, N,{ }^{*}\right)$ is a fuzzy normed space on $X \times Y$.

Let $\left(X,\|\|,. L_{1}, R_{1}\right)$ and $\left(Y,\|\|,. L_{2}, R_{2}\right)$ be two fuzzy normed spaces by the above definition, where $R_{1}(x, y) \leq \max (x, y)$, and $R_{2}(x, y) \leq \max (x, y)$. Then $(X \times Y,\|\cdot\|, L, R)$ is a fuzzy normed space where $R=\max$ and $L=0$,

$\|(x, y)\|(t)=\left\{\begin{array}{l}1, \\ \max \{\|x\|(t),\|y\|(t)\},\end{array}\right.$

$t \in\left[\min \left\{\|x\|_{1}^{-}, \|\left. y\right|_{1} ^{-}\right\}, \max \left\{\|x\|_{2}^{+},\|y\|_{2}^{+}\right\}\right]$,

otherwise.

By applying the induction method, we conclude that the Cartesian product of fuzzy normed spaces, $X_{1} \times X_{2} \times \ldots \times X_{n}$ is also fuzzy normed space. We have also same result in Menger probabilistic spaces.

Let $(X,\|\|$.$) be a classical real normed space. Felbin$ and Samanta have been shown that classical real normed space can be considered as fuzzy normed linear spaces (Felbin's type and Samanta's type). In the following we bring an example of a fuzzy normed space which is not a real normed space in the classical sense. Therefore the spectrum of the category of fuzzy normed linear spaces is broader than the category of normed spaces. This is why the study of fuzzy normed linear spaces (Felbin's type and Samanta's type) is of great importance.

Let $\Omega$ be a nonempty open set in some euclidean space. It is well known that $\Omega$ is the union of countably sets $K_{n} \neq$ $\varnothing$, which can be chosen so that $K_{n}$ lies in the interior of $K_{n+1}$ $(n=1,2,3, \ldots)$. The linear space $C(\Omega)$, is the vector space 
of all complex valued continuous functions on $\Omega$, topologized by the family of non-decreasing classical seminorms $p_{n}(f)=\sup \left\{|f(x)| ; x \in K_{n}\right\}$. In the following results, we will prove that $C(\Omega)$ is fuzzy normable but it is not so in classical analysis, where $\Omega \subset \Re^{n}$ is an open subset.

Theorem: The linear space $C(\Omega)$ is fuzzy normable in general. For $\alpha \in(0,1]$, there exists $n \in \mathrm{N}$ such that $\frac{1}{n+1}<\alpha \leq \frac{1}{n}$. Let $f \in C(\Omega)$ define $f_{\alpha}^{-}=\sup \left\{|f(x)| ; x \in K_{1}\right\}$ and $f_{\alpha}^{+}=\sup \left\{|f(x)| ; x \in K_{n+1}\right\}$. Then $\left\{\left[f_{\alpha}^{-}, f_{\alpha}^{+}\right] ; \alpha \in(0,1]\right\}$ is a family of nested bounded closed intervals. We define the fuzzy norm map $\|$.$\| on C(\Omega)$ as:

$\forall t \in \mathfrak{R},\|f\|(t)=\sup \left\{\alpha \in(0,1] ; t \in\left[f_{\alpha}^{-}, f_{\alpha}^{+}\right]\right\}$.

It is easy to see that for $f \in C(\Omega),\|f\|$ is a fuzzy real number. Now we show that $(C(\Omega),\|\|,. \min , \max )$ is a fuzzy normed space. Also by definition

$$
\rho_{n}(f, t)= \begin{cases}0, & t \leq \rho_{n}(f), \\ 1, & t>\rho_{n}(f) .\end{cases}
$$

We have shown that that $\rho_{n}(f, t)$ is a fuzzy seminorm for all $n \in N$ and $P=\left\{\rho_{n} ; n \in N\right\}$ is the separating family of seminorms on $C(\Omega)$. According to the above theorem we can define a fuzzy norm on $C(\Omega)$ as

$$
N(f, t)=d(f, 0, t)=\sum_{i=1}^{\infty} 2^{-i} \rho_{n}(f, t) .
$$

Then, $(C(\Omega), N)$ is a fuzzy normed space. But $C(\Omega)$ is not normable in classical analysis, where $\Omega$ is an open subset of $\mathfrak{R}^{n}$.

Furthermore, $C(\Omega) \times C(\Omega)$ is also a Fuzzy Banach space with the norm where $(f, g) \in C(\Omega) \times C(\Omega)$ and $t \in \mathfrak{R}$ defined by

and

$$
N((f, g), t)=\min \{N(f, t), N(g, t)\},
$$

$$
\|(f, g)\|(t)=\left\{\begin{array}{l}
1, \\
\max \{\|f\|(t),\|g\|(t)\},
\end{array}\right.
$$

$t \in\left[\min \left\{\|f\|_{\alpha}^{-}, \|\left. g\right|_{\alpha} ^{-}\right\}, \max \left\{\|f\|_{\alpha}^{+},\|g\|_{\alpha}^{+}\right\}\right]$,

otherwise.

Definition: [Wu and Ma (1991)] let $\eta$ be a fuzzy number in $\Re(I)$ and we write $\eta^{-}(\alpha)=\eta_{\alpha}^{-}$and $\eta^{+}(\alpha)=\eta_{\alpha}^{+}$as the function of $\alpha \in[0,1]$, then the function

$\pi: \mathfrak{R}(I) \rightarrow C(\Omega) \times C(\Omega)$ defined by

$\pi(\eta)=\left(\eta^{-}(\alpha), \eta^{+}(\alpha)\right)$ is injective. Now, we prove that each element in $\mathfrak{R}(I)$ can be identified with an element $\left(\eta^{-}(\alpha), \eta^{+}(\alpha)\right)$ in $C(\Omega) \times C(\Omega)$, where $\eta^{-}(\alpha)=\eta_{\alpha}^{-}$and $\eta^{+}(\alpha)=\eta_{\alpha}^{+}$, and this identification is isometric and isomorphic.

An important problem in topology is embedding problem. Theorems asserting the embedding of a space into some other space which is more manageable than the original space are known as embedding theorems.

Theorem: (Embedding Theorem). The function $\pi: \Re(I) \rightarrow C(\Omega) \times C(\Omega)$ be defined by $\pi(f)=\left(f^{+}(\alpha), f^{-}(\alpha)\right)$, if $\alpha>0.5$, and $\left(f^{-}(\alpha), f^{+}(\alpha)\right)$, if $\alpha<0.5$. Then, the following properties hold true:

(i) $\pi$ is injective.

(ii) $\pi\left(\left(1_{\{s\}} \otimes f_{1}\right) \oplus\left(1_{\{t\}} \otimes f_{2}\right)\right)=s \pi\left(f_{1}\right)+t \pi\left(f_{2}\right)$

for all $\eta_{11} \eta_{2} \in \mathfrak{R}(I), s>0$ and $t>0$.

(iii) $d_{\{F\}}\left(f_{1}, f_{2}\right)(t)=N\left(\pi\left(f_{1}\right)-\pi\left(f_{2}\right), t\right)=$ $\sup \left\{\alpha \in(0,1], \quad t \in\left[f_{\alpha}^{-}, f_{\alpha}^{+}\right]\right\}$. That is to say, $\mathfrak{R}(I)$ can be embedded into $C(\Omega) \times C(\Omega)$ isometrically and isomorphically.

Proof: (i): Let $f_{1}, f_{2}$ be two distinct fuzzy numbers such that $\pi\left(f_{1}\right)=\pi\left(f_{2}\right)$. Then $\left(f_{1 \alpha}^{-}, f_{1 \alpha}^{+}\right)=\left(f_{2 \alpha}^{-}, f_{2 \alpha}^{+}\right)$, i.e.

$\left(f_{1}^{-}(\alpha), f_{1}^{+}(\alpha)\right)=\left(f_{2}^{-}(\alpha), f_{2}^{+}(\alpha)\right)$. Then

$f_{1}^{-}(\alpha)=f_{2}^{-}(\alpha)$, and $f_{1}^{+}(\alpha)=f_{2}^{+}(\alpha)$, because the two real open intervals are equal which contradicts our assumption and consequently the mapping $\pi$ is injective. (ii): By the definition of fuzzy number and function of $\pi$ we have:

$$
\begin{aligned}
& \pi\left(\left(1_{\{s\}} \otimes f_{1}\right) \oplus\left(1_{\{t\}} \otimes f_{2}\right)\right)=\left(\left[\left(1_{\{s\}} \otimes f_{1}\right) \oplus\left(1_{\{t\}} \otimes f_{2}\right)\right]_{\alpha}^{-},\left[\left(1_{\{s\}} \otimes f_{1}\right) \oplus\left(1_{\{t\}} \otimes f_{2}\right)\right]_{\alpha}^{+}\right)= \\
& \left(\left[\left(1_{\{s\}} \otimes f_{1}\right)\right]_{\alpha}^{-} \oplus\left[\left(1_{\{t\}} \otimes f_{2}\right)\right]_{\alpha}^{-},\left[\left(1_{\{s\}} \otimes f_{1}\right)\right]_{\alpha}^{+} \oplus\left[\left(1_{\{t\}} \otimes f_{2}\right)\right]_{\alpha}^{+}\right)= \\
& \left.\left.\left(\left[\left(1_{\{s\}}\right]_{\alpha}^{-} \otimes\left[f_{1}\right)\right]_{\alpha}^{-} \oplus\left[\left(1_{\{t\}}\right]_{\alpha}^{=} \otimes\left[f_{2}\right)\right]_{\alpha}^{-},\left[\left(1_{\{s\}}\right]_{\alpha}^{+} \otimes f_{1}\right)\right]_{\alpha}^{+} \oplus\left[\left(1_{\{t\}}\right]_{\alpha}^{+} \otimes f_{2}\right)\right]_{\alpha}^{+}\right)= \\
& \left.\left.\left.\left(\left(s\left[f_{1}\right)\right]_{\alpha}^{-} \oplus t\left[f_{2}\right)\right]_{\alpha}^{-}, s\left[f_{1}\right)\right]_{\alpha}^{+} \oplus t\left[f_{2}\right)\right]_{\alpha}^{+}\right)=s \pi\left(f_{1}\right)+t \pi\left(f_{2}\right) .
\end{aligned}
$$

(iii): $d_{\{F\}}\left(f_{1}, f_{2}\right)=\sup _{0<\alpha<1} \max \left\{\left|f_{1 \alpha}^{-}-f_{2 \alpha}^{-}\right|,\left|f_{1 \alpha}^{+}-f_{2 \alpha}^{+}\right|\right\}=\max \left[\sup _{0<\alpha<1}\left|f_{1 \alpha}^{-}-f_{2 \alpha}^{-}\right|,\left|f_{1 \alpha}^{+}-f_{2 \alpha}^{+}\right|\right]=$ $=\max \left\{\sup \left\{\alpha \in(0,1] \quad t \in\left[f_{1 \alpha}^{-}, f_{1 \alpha}^{+}\right], \sup \left\{\alpha \in(0,1] \quad t \in\left[f_{2 \alpha}^{-}, f_{2 \alpha}^{+}\right]\right\}\right\}=\max \left\{\left\|\pi\left(f_{1}\right)\right\|(t),\left\|\pi\left(f_{2}\right)\right\|(t)\right\}\right.$ $=\left\|\pi\left(f_{1}\right), \pi\left(f_{2}\right)\right\|(t)=N\left(\pi\left(f_{1}\right)-\pi\left(f_{2}\right), t\right)$. 
Therefore, we proved that the set of all fuzzy numbers can be embedded into a Fuzzy Banach space isometrically and isomorphically. Inspired by this specific Banach space, a fuzzy optimization problem can be transformed into a multiobjective optimization problem which can be solved by using interactive fuzzy decision making procedure. Under this setting, the fuzzy optimization problem is transformed into a biobjective programming problem by applying the embedding theorem and the optimal solution of the crisp optimization problem obtained from the fuzzy optimization problem is also an optimal solution of the original fuzzy optimization problem under the set of core values of fuzzy numbers.

Now, we consider a class of optimization problems which have multiple objective functions subject to a set of fuzzy relation equations.

The general form of a Banach application to fuzzy logic portfolio selection model can be described as follows:

$$
\text { maximize } Z=r_{1} x_{1} \oplus r_{2} x_{2} \ldots \oplus r_{n} x_{n}
$$

such that $\tilde{a}_{1} x_{1} \oplus \tilde{a}_{2} x_{2} \oplus \ldots \widetilde{a}_{n} x_{n} \oplus \leq\left(\widetilde{b}_{1}, \widetilde{b}_{2}, \ldots, \widetilde{b}_{n}\right)$,

$$
x_{i} \geq 0 \quad(i=1,2, \ldots, n) \text {. }
$$

A more approximative style to implement a numerical solution to a portfolio selection is described as an application such as in Eqn. (2) or in Eqn. (3). In the following section we present optimization models which illustrate the solution to multiobjective portfolio selection models in a fuzzy optimization environment.

$$
\operatorname{maximize} E\left[\sum_{i=1}^{n} r_{i} x_{i}\right]
$$$$
\text { and minimize } \operatorname{Var}\left[\sum_{i=1}^{n} r_{i} x_{i}\right]
$$

such that $\sum_{i=1}^{n} x_{i}=1, \quad x_{i} \geq 0 \quad(i=1,2, \ldots, n)$;

$$
\operatorname{maximize} \quad Z_{r}=E\left[\sum_{i=1}^{n} r_{i} x_{i}\right]
$$

and minimize $Z_{v}=\operatorname{Var}\left[\sum_{i=1}^{n} r_{i} x_{i}\right]=\sum_{i=1}^{n} x_{i}^{2} \operatorname{Var}\left(r_{i}\right)+2 \sum_{1 \leq i \leq j \leq n} x_{i} x_{j} \operatorname{Cov}\left(r_{i}, r_{j}\right)$

$$
\text { such that } \sum_{i=1}^{n} x_{i}=1, \quad x_{i} \geq 0 \quad(i=1,2, \ldots, n)
$$

\section{FNLP technique for solution of Multiobjective Programming Problem (MONLP)}

Consider the following general Multiobjective Nonlinear Programming (MONLP) optimization problem:

$$
\text { minimize }\left(f_{1}(x), f_{2}(x), \ldots, f_{k}(x)\right)^{T}
$$

such that $x \in X=\left\{x \in R_{+}^{n} ; g_{j}(x) \leq 0(j=1,2, \ldots, m)\right\}$, $l_{i} \leq x_{i} \leq u_{i}(i=1,2, \ldots, n)$
Step 1: In order to solve the MONLP problem in (12) we solve the problem as only one objective while disregarding the other ones. The procedure is successively repeated to find the ideal solution.

Step 2: Once all optimal solutions for each objective are solved, a pay-off matrix for each single objective can be obtained. The obtained pay-off matrix is described as follows:

$$
\begin{aligned}
& f_{1}(x) \quad f_{2}(x) \quad \cdots \quad f_{k}(x) \\
& x^{1} \quad\left[\begin{array}{llll}
f_{1}^{*}\left(x^{1}\right) & f_{2}\left(x^{1}\right) & \cdots & f_{k}\left(x^{1}\right)
\end{array}\right] \\
& \begin{array}{lllll}
x^{2} & f_{1}\left(x^{2}\right) & f_{2}^{*}\left(x^{2}\right) & \cdots & f_{k}\left(x^{2}\right)
\end{array} .
\end{aligned}
$$

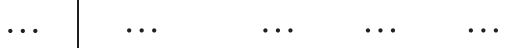

$$
\begin{aligned}
& x^{k}\left[\begin{array}{llll}
f_{1}\left(x^{k}\right) & f_{2}\left(x^{k}\right) & \cdots & f_{k}^{*}\left(x^{k}\right)
\end{array}\right]
\end{aligned}
$$

Optimal solutions for each objective function $f_{1}(x), f_{2}(x), \ldots, f_{k}(x)$ are given as $x^{1}, x^{2}, \ldots, x^{k}$. From this computation we derive the upper and lower bounds of each objective function $f_{n}(x) \quad(n=1,2, \ldots, k)$. The upper and lower bounds are given in the following says:

$$
\begin{aligned}
& U_{n}=\max \left\{f_{n}\left(x_{1}\right), \quad f_{n}\left(x_{2}\right), \cdots, f_{n}\left(x_{k}\right)\right\}, \\
& L_{n}=\max \left\{f_{n}\left(x_{1}\right), \quad f_{n}\left(x_{2}\right), \cdots, f_{n}\left(x_{k}\right)\right\} \text {. }
\end{aligned}
$$

Step 3: Fuzzy set: characteristic function $\mu_{n}$ of crisp set to each member in $X$ with $\mu_{n}: X \rightarrow[0,1]$. The assigned values is called a membership function and the set $A=\left\{\left(x, \mu_{n}(x)\right): x \in X\right\}$, defined by $\mu_{n}(x)$ for $x \in X$ is called afuzzy set. Membership function, crisp problem the membership function $\mu_{k}$ and $v_{k}$ are used respectively to solve the maximization of objective function $f_{1}(x)$ and minimization of objective function $f_{2}(x)$. We note:

$$
\begin{gathered}
\mu_{k}\left(Z_{k}(\bar{X})\right)=\left\{\begin{array}{cl}
1, & \text { if } Z_{k}(\bar{X}) \leq L_{k}, \\
\frac{U_{k}-Z_{k}(\bar{X})}{U_{k}-L_{k}}, & \text { if } L_{k} \leq Z_{k}(\bar{X}) \leq U_{k}, \\
0, & \text { if } Z_{k}(\bar{X}) \geq U_{k},
\end{array}\right. \\
v_{k}\left(Z_{k}(\bar{X})\right)=\left\{\begin{array}{cl}
1, & \text { if } Z_{k}(\bar{X})>U_{k}, \\
\frac{Z_{k}(\bar{X})-L_{k}}{U_{k}-L_{k},} & \text { if } L_{k} \leq Z_{k}(\bar{X}) \leq U_{k}, \\
0, & \text { if } Z_{k}(\bar{X}) \leq L_{k} .
\end{array}\right.
\end{gathered}
$$

With $w_{1}$ and $w_{2}$ being crisp weights for portfolio returns and portfolio variance, then the previous model can be formulated as follows:

maximize $\alpha$ such that $w_{1} \circ\left(\frac{Z_{r}-Z_{r}^{l}}{Z_{r}^{u}-Z_{r}^{l}}\right) \geq \alpha$,

$$
\begin{aligned}
& w_{2} \circ\left(\frac{Z_{v}^{u}-Z_{v}}{Z_{r}^{u}-Z_{r}^{l}}\right) \geq \alpha, \\
& \sum_{i=1}^{n} x_{i}=1, \quad 0 \leq \alpha \leq 1, \quad w_{1}+w_{2}=1,
\end{aligned}
$$


where $»^{0} \ll$ is the composition operator for a function $\mathrm{w}$, and a multiplication symbol for our applied exanple in the following section.

Step 4: Solve crisp nonlinear programming to get optimal solution of the MONLP by application of various proposed well-known methods.

Crisp Weights:

Crisp relative weights measure the preferences for objective goals and reflect their relative importance. The positive crisp weights $w_{1}(i=1,2, . ., m)$. for the crisp model are normalised as $\sum_{i=1}^{m} w_{i}=1$. In alternative scenarios we propose different weights to reflect investors alternative preferences. Hence, a higher weight value gives evidence for more importance of the objective goal. In our fuzzy non-linear programming models, we tested various related scenarios.

\section{Fuzzy Weighted Model:}

$$
\begin{gathered}
\text { maximize } \alpha \text { such that } \mu_{w_{1}} \circ\left(\frac{Z_{r}-L_{k}}{U_{k}-L_{k}}\right) \geq \alpha, \\
v_{w_{2}} \circ\left(\frac{U_{k}-Z_{v}}{U_{k}-L_{k}}\right) \geq \alpha, \\
\sum_{i=1}^{n} x_{i}=1, \quad 0 \leq \alpha \leq 1 .
\end{gathered}
$$

\section{Numerical Illustration}

This section gives an illustrative example to present optimal solutions to MONLP models. We selected six stock exchange shares which are traded in the Frankfurt DAX stock exchange market. The daily data ranges between January 2011 and January 2014. We selected each share from different sectors to account for alternative portfolio investments. Table 1 shows expected return and variance of the alternative shares. The companies are as follows: Deutsche Bank (DB), Daimler AG (DM), Henkel AG (HK), E.ON. (EON), BASF AG (BASF), Allianz AG (AL).

The covariance matrix for the selected data sample is given in Table 2.

In Table 3, the pay-off matrix indicates the optimal results of each single objective.

Weighted Portfolio Selection:

Table 4 shows the optimal solution values according the relative investor preferences.

Finally, we derive the Fuzzy weighted model solution in Table 5.

\section{Conclusion}

The optimal solution of the crisp optimization problem deduced from the fuzzy optimization problem by using embedding theorem is an optimal solution of the original fuzzy optimization problem under the set of core values of fuzzy real numbers. In a portfolio selection problem, the expected return, risk, liquidity cannot be predicted accurately. So the investor generally makes his portfolio choice according to his knowledge and his economic wisdom. Thus, deterministic portfolio selection is not a sensible option for the investor. Because of the existence of several non-stochastic factors in a stock market, fuzzy portfolio selection models have been proposed by model within fuzzy

Table 1: Expectation and variance for selected stocks (in growth).

\begin{tabular}{lcccccc} 
& DB & DM & HK & EON & BASF & AL \\
\hline $\begin{array}{l}\text { Expected } \\
\text { Return }\end{array}$ & 1.00 & 1.00 & 1.00 & 0.99 & 1.00 & 1.00 \\
\hline Variance & 0.00061 & 0.000395683 & 0.000181948 & 0.000348582 & 0.000289541 & 0.000360242 \\
\hline
\end{tabular}

Table 2: Covariance matrix.

\begin{tabular}{ccccccc} 
& DB & DM & HK & EON & BASF & AL \\
\hline DB & 0.22349517 & 0.223058336 & 0.223346256 & 0.222815933 & 0.222994258 & 0.22296937 \\
\hline DM & 0.223058336 & 0.223449288 & 0.223118822 & 0.222894143 & 0.223114491 & 0.223061775 \\
\hline HK & 0.223346256 & 0.223118822 & 0.223689574 & 0.222889422 & 0.223063648 & 0.223015952 \\
\hline EON & 0.222815933 & 0.222894143 & 0.222889422 & 0.222987418 & 0.222826304 & 0.222803293 \\
\hline BASF & 0.222994258 & 0.223114491 & 0.223063648 & 0.222826304 & 0.223347778 & 0.222988255 \\
\hline AL & 0.22296937 & 0.223061775 & 0.223015952 & 0.222803293 & 0.222988255 & 0.222985648 \\
\hline
\end{tabular}


Table 3: Pay-off matrix signifying optima.

\begin{tabular}{ccc} 
& $\mathrm{Zr}$ & ZVar \\
\hline (DB,DM,HK,EON,BASF,AL)1 & 1.000994 & $0.1885714 \mathrm{E}-03$ \\
\hline (DB,DM,HK,EON,BASF,AL)2 & $0.1885714 \mathrm{E}-03$ & 1.000994 \\
\hline
\end{tabular}

Note. The model is solved as in Step 2. The optimal values are obtained by iteratively solving and combining the single objective functions Zr and ZVar.

Table 4: Relative preferences of the investors.

\begin{tabular}{cccccc} 
Case & W1 & W2 & SOLRETURN & SOLVAR & ALPHA \\
\hline 1 & 0.5 & 0.5 & 1.001174 & 0.000000 & 0.5000942 \\
\hline 2 & 0.6 & 0.4 & 0.6675093 & 0.000000 & 0.4000754 \\
\hline 3 & 0.4 & 0.6 & 1.501671 & 0.000000 & 0.6001131 \\
\hline
\end{tabular}

Note. The abbreviations are as follows: w1, weight for return maximizing objective, w2, weight for variance minizing objective, SOLRETURN, solution value for return objective, SOLVAR, solution value for variance objective, alpha, solution value for final multiobjective optimal solution.

Table 5: Optimal solutions in the fuzzy weighted model.

\begin{tabular}{cccccccc} 
Case & FW1 & FW2 & SOLRETURN & SOLVAR & ALPHA & W1 & W2 \\
\hline 1 & {$[0.4,0.5]$} & {$[0.6,0.7]$} & 1.667149 & 0.000000 & 0.7001319 & 0.5000000 & 0.7000000 \\
\hline 2 & {$[0.6,0.7]$} & {$[0.4,0.5]$} & 1.261565 & 0.000000 & 0.5000942 & 0.7000000 & 0.5000942 \\
\hline 3 & {$[0.5,0.6]$} & {$[0.7,0.8]$} & 1.470387 & 0.000000 & 0.8001507 & 0.6000000 & 0.8000000 \\
\hline 4 & {$[0.7,0.8]$} & {$[0.5,0.6]$} & 1.168285 & 0.000000 & 0.6001131 & 0.8000000 & 0.6000000 \\
\hline
\end{tabular}

Note. See Table 4. FW1 and FW2 denote the fuzzy weights.

norm. This paper extends the classical Banach spaces into fuzzy Banach and Menger probablistic Banach spaces model in fuzzy environment. This embedding theorem can be used for multiobjective decision making and has successfully been applied to optimization problems. We tried out various scenarios for portfolio selection which are pointing out different investor preferences. In Case 1 (See Table 4), the investor treats both objectives equally. In Case 2, the investor put more weight on portfolio return. In Case 3 , the investor prefers to put more weight on the portfolio variance. We observe that different investor preferences determine the optimal solution to multiobjective fuzzy portfolio selection models according to alternative scenarios.

\section{Literature}

Bag, T., \& Samanta, T. K. (2003). Finite dimensional fuzzy normed linear spaces. J. Fuzzy Math. 11(3), 687-705.
Bag, T., \& Samanta, T. K. (2008). A comparative study of fuzzy norms on a linear space. Fuzzy Sets and Systems, 159, 670684, http://dx.doi.org/10.1016/j.fss.2007.09.011

Bermúdeza, J.D., Segurab, J.V., \& Verchera, E. (2012). A multiobjective genetic algorithm for cardinality constrained fuzzy portfolio selection. Fuzzy Sets and Systems, 188, 16-26, http://dx.doi.org/10.1016/j.fss.2011.05.013

Bilbao-Terol, A., Arenas-Parra, M., Canal-Fernandez, V. (2012). Selection of Socially Responsible Portfolios using Goal Programming and fuzzy technology, Information Sciences, 189, 110-125, http://dx.doi.org/10.1016/j.ins.2011.12.001

Bhattacharyya R., \& Kar S. (2011). Multiobjective fuzzy optimization for portfolio selection: an embedding theorem approach. Turkish Journal of Fuzzy Systems, 2(1), 14-35. Retrieved April 3, 2014, from http://www.tjfs-journal.org/TJFS_v2n1_ pp14_35.pdf

Cheng, S.C., \& Mordeson, J.N. (1994). Fuzzy linear operator and fuzzy normed linear spaces. Bull. Cal. Math. Soc. 86, 429-436. 
Felbin, C. (1992). Finite dimensional fuzzy normed linear spaces. Fuzzy Sets and Systems, 48, 239-248, http://dx.doi. org/10.1016/0165-0114(92)90338-5

Gupta, P., Inuiguchi, M., Mehlawat, M. K., \& Mittal, G. (2012), Multiobjective credibilistic portfolio selection model with fuzzy chance-constraints. Information Sciences, 229, 1-17, http://dx.doi.org/10.1016/j.ins.2012.12.011

Huang, X. \& Qiao, L. (2012). A risk index model for multiperiod uncertain portfolio selection. Information Sciences, 217, 108-116, http://dx.doi.org/10.1016/j.ins.2012.06.017

Kaleva, O., \& Seikkala, S. (1984). On fuzzy metric spaces. Fuzzy Sets and Systems, 12, 215-229, http://dx.doi. org/10.1016/0165-0114(84)90069-1

Katsaras, A.K. (1984). Fuzzy topological vector spaces II, Fuzzy Sets and Systems, 12, 143-154, http://dx.doi.org/10.1016/01650114(84)90034-4

Khalili-Damghani, K., Sadi-Nezhad, S., Hosseinzadeh Lotfi, F., \& Tavana M. (2013). A hybrid fuzzy rule-based multicriteria framework for sustainable project portfolio selection, Information Sciences, 220, 442-462, http://dx.doi. org/10.1016/j.ins.2012.07.024

Kramosil, I. and Michalek, J. (1975). Fuzzy metric and statistical metric spaces, Kybernetica, 11, 326-334.

Li, J., \& Xu, J. (2013). Multiobjective portfolio selection model with fuzzy random returns and a compromise approach-based genetic algorithm, Information Sciences, 220, 507-521, http:// dx.doi.org/10.1016/j.ins.2012.07.005

Liu, Y.-J., Zhang, W.-G., \& Xu, W.-J. (2012). Fuzzy multiperiod portfolio selection optimization models using multiple criteria, Automatica, 48, 3042-3053, http://dx.doi.org/10.1016/j. automatica.2012.08.036

Markowitz, H., (1952). Portfolio selection. Journal of Finance, 7, 77-91. Retrieved from http://www.jstor.org/stable/2975974

Menger K. (1942). Statistical metrics, Proc. Nat. Acad. Sci. USA, 28, 535-7.

Metaxiotis K., \& Liagkouras K., (2012). Multiobjective Evolutionary Algorithms for Portfolio Management: A comprehensive literature review, Expert Systems with Applications, 39, 11685-11698, http://dx.doi.org/10.1016/j. eswa.2012.04.053

Sadeqi I., \& Solatikia F. (2010). The category of fuzzy normed linear spaces, J. Fuzzy Math., 18, 733-742.

Sherstnev A.N. (1962), Random normed spaces: Problems of completeness (in Russian). Kazan. Gos. Univ. Ucen. Zap., 122, 3-20.

Rebiasz, B. (2013). Selection of efficient portfolios-probabilistic and fuzzy approach, comparative study. Computers \& Industrial Engineering, 64, 1019-1032, http://dx.doi. org/10.1016/j.cie.2013.01.011

Tsaur, R.-C. (2013). Fuzzy portfolio model with different investor risk attitudes, European Journal of Operational Research, 277(2), 385-390, http://dx.doi.org/10.1016/j.ejor.2012.10.036
Weber G.-W., \& Solatikia F. (2013). Generalization by Optimization in Menger Probabilistic Normed Spaces with Application on Portfolio in Insurance (Editorial). J. Appl. Computat. Math., 2(3), 1000e131, http://dx.doi.org/10.4172/2168$9679.1000 \mathrm{e} 131$

Wu C. X. \& Ma M. (1991). Embedding Problem of Fuzzy Number Space: Part I, Fuzzy Sets and Systems, 44, 33-38.

Wu, H.-C. (2004). Evaluate Fuzzy Optimization Problems Based on Biobjective Programming Problems, Computers and Mathematics with Applications 47, 893-902, http://dx.doi. org/10.1016/S0898-1221(04)90073-9

Zadeh L.A. (1965). Fuzzy sets. Information and Control, 8, 338353, http://dx.doi.org/10.1016/S0019-9958(65)90241-X

Farnaz Solatikia is a Ph.D. student and Teaching Assistant in at the Department of Engineering Science and Research Assistant in Graduate School of Applied Mathematics in METU, Ankara. She has specialized on applied mathematics in fuzzy environment. Farnaz has received MSc. in Mathematics, on the Faculty of Basic Sciences. Her research interests include Fuzzy Mathematics, Functional Analysis, portfolio optimization, Applied Probability, and differential equation models. She has some publications in journals and conferences.

Erdem Kilic has a Ph.D. in financial economics with emphasis on financial econometrics and financial modeling. $\mathrm{He}$ is an assistant professor at the Department of Economics, MEF University in Istanbul. His main research interests include financial economics and stochastic models in finance. Recently, he has been focusing on optimal contracts under asymmetric information, multiobjective portfolio selection models, and effects of consumer confidence dynamics on business activity.

Gerhard-Wilhelm Weber is a professor at Institute of Applied Mathematics, METU, Ankara, Turkey. His research in on optimization and control, OR, financial mathematics, life and human sciences, data mining and inverse problems. G.-W. Weber received Diploma and Doctorate in Mathematics and Economics/BA, at RWTH Aachen, and Habilitation at TU Darmstadt. He held Professorships by proxy at University of Cologne, and TU Chemitz (Germany); he has several international affiliations, memberships and honorary positions, and is »Advisor to EURO Conferences«. 